\title{
Magnetic Cataclysmic Variables
}

\author{
B. Kalomeni \\ University of Ege, Department of Astronomy \& Space Sciences, 35100, Bornova-İzmir, Turkey \\ Department of Physics, İzmir Institute of Technology, 35430, Urla-İzmir, Turkey
}

\begin{abstract}
We present a study on magnetic cataclysmic variables (polars) using the collected data from the literature. These parameters (mass, radius, magnetic field intensity, mass transfer rate, etc.) and the relations between them are important to understand their nature, to explain the observed properties of them and to test the current evolutionary models.
\end{abstract}

Keywords: cataclysmic variables, polars, stellar evolution

PACS: $97.80 . \mathrm{Gm}, 97.20 . \mathrm{Qt}$

\section{INTRODUCTION}

Polars are interacting binary systems consisting of a magnetic white dwarf (WD) and a usually late type main-sequence star. In a binary system when the secondary fills its Roche-lobe either by nuclear evolution or by angular momentum loss the mass transfer starts. In polars mass transfer between the components occurs by removing angular momentum, mainly driven by magnetic braking and by gravitational radiation. Observed mass accretion rates in polars are relatively low in comparison with the binaries consisting of non-degenerate components (see [1]). Occasionally, mass transfer between the components either reduces or ceases. This state of polars can last from days to years (see [2], [1]).

WDs in polars provide a natural laboratory to study the physics under high magnetic fields. In addition, studies on the origin and evolution of the magnetic field in WDs may bear important information on its role in stellar evolution, as well as the field distribution of WDs in a binary system (see [3]). Most of the important observational variations in polars should be due to the strong magnetic fields in WDs. We have compiled the data of more than 90 polars. The more detailed study and the data can be found in [4].

\section{PROPERTIES OF POLARS}

The orbital periods of polars ranges between $\sim 1 \mathrm{hr}$ to $\sim 8 \mathrm{hr}$. The number of cataclysmic variables (CVs) with orbital periods between 2 and $3 \mathrm{hr}$ were less than short and long-period polars. Advances in photometric, spectroscopic, and space-based observations made possible the detection of faint systems. The period gap, therefore, seems to be filling up with newly discovered CVs [5]. In Fig. 1a the orbital period distribution of polars is shown. More than a quarter of the polars are found in the period gap while the number of polars peak at orbital periods between 1.5-2 $\mathrm{hr}$ (see also [6]). Hameury et al. (1988) discussed that as systems evolve from higher to lower periods they spend longer time at the edge of the lower period gap. Bright CVs, that are favorable targets to discover and observe, are though to have long orbital periods [7], because of the higher accretion rates and more luminous donor stars [8]. The number of polars below the period gap, however, is higher than the systems above the period gap.

Magnetic WDs on average appear to be massive than non-magnetic WDs indicating more massive and younger ancestor star[9]. For the origin of the strong magnetic fields in WDs Tout et al. (2008)[3] proposed a magnetic dynamo operating during common envelope evolution. Core merging in a common envelope proposed to result in a single highly magnetic WD[3]. Magnetic field intensity is a crucial parameter since it determines most of the observed properties of polars. The magnetic field intensities of polars range from $\sim 11 \mathrm{MG}$ to $\sim 235 \mathrm{MG}$. Most of these field strengths have been determined by cyclotron humps that are important in determination of the system's geometry (see [10]). The magnetic fields of $\sim 70 \%$ of the WDs are between $15 \mathrm{MG}-54 \mathrm{MG}$ (Fig. 1b). A small number of polars with relatively strong magnetic fields have been discovered. It is possibly because the evolution time scale of them are shorter than the systems with lower field intensities due to strong angular momentum loss ([11] and references therein). The masses of the WDs in polars ranges between $0.4 M_{\odot}-1 M_{\odot}$ with a mean mass of $0.77 M_{\odot}$ [12]. Mass distribution of WDs as a function of orbital period is shown in Fig. 2a. Low mass WDs appear to be in the period 
gap while on either side massive WDs are observed. Magnetic field intensity and masses of the WDs in polars show no remarkable correlation (Fig. 2b). In Fig. 2c the spectral type of secondaries in polars $v s$ orbital period is shown. Long-period polars have early-spectral types than short-period systems.
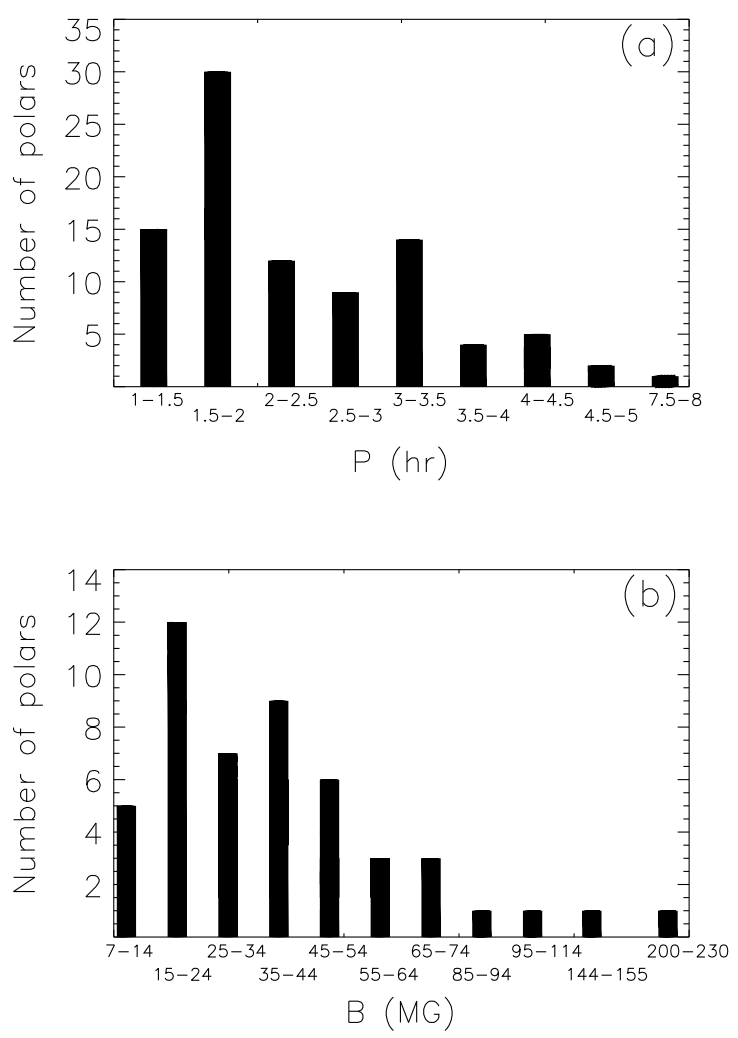

FIGURE 1. (a) Distribution of orbital periods for AM Her binaries and (b) the magnetic field of primary accretion column in polars.
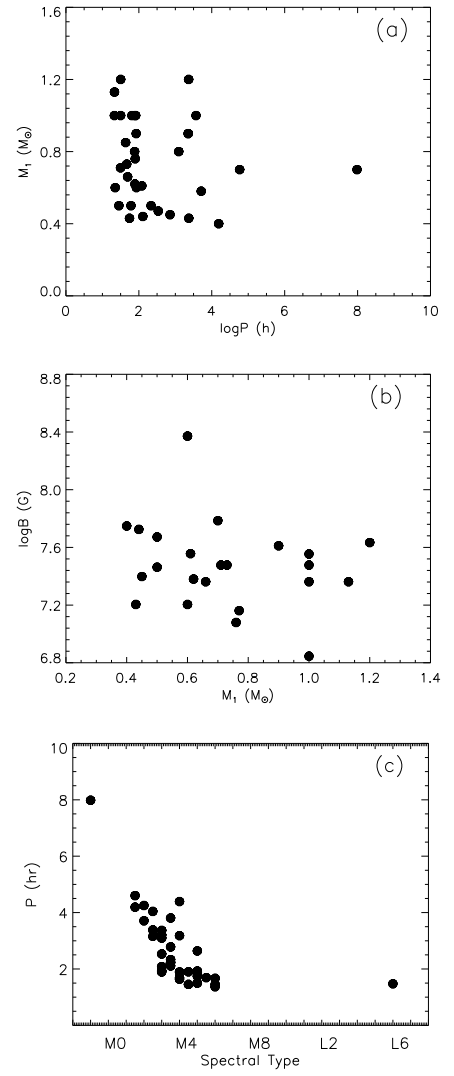

FIGURE 2. (a) WD mass as a function of orbital period, (b) magnetic field strength vs. WD mass, (c) orbital period as a function of spectral type of secondaries.

Polars not only provide a way to study the physics of magnetic WDs but also bear important information on the nature and evolution of low-mass main-sequence stars. Therefore, in numerous studies the general properties and the evolution of mCVs have been considered over the last four decades (e.g. [13], [11]).

Acknowledgments We acknowledge the financial support from TÜBİTAK (Project No:109T047) and EBİLTEM.

\section{REFERENCES}

1. B. Kalomeni, and K. Yakut, $A J$ 136, 2367-2372 (2008), 0811.0568.

2. K. Wu, and L. L. Kiss, A\&A 481, 433-439 (2008), 0802.0019.

3. C. A. Tout, D. T. Wickramasinghe, J. Liebert, L. Ferrario, and J. E. Pringle, MNRAS 387, 897-901 (2008), 0805 . 0115.

4. B. Kalomeni, et al., in preparation (2011).

5. N. A. Katysheva, and E. P. Pavlenko, Astrophysics 46, 114-128 (2003).

6. J. M. Hameury, A. R. King, J. P. Lasota, and H. Ritter, MNRAS 231, 535-547 (1988).

7. J. Southworth, C. M. Copperwheat, B. T. Gänsicke, and S. Pyrzas, A\&A 510, A100+ (2010), 0912.0156.

8. C. M. Copperwheat, T. R. Marsh, and V. S. Dhillon, et al., MNRAS 402, 1824-1840 (2010).

9. I. Suh, and G. J. Mathews, ApJ 530, 949-954 (2000), arXiv: astro-ph/9906239.

10. B. Kalomeni, E. R. Pekünlü, and K. Yakut, $A \& A$ 439, 823-833 (2005).

11. M. Cropper, SSRv 54, 195-295 (1990).

12. B. Kalomeni, "Angular Momentum Loss in Polars," in IAU Symposium, edited by G. Bruzual \& S. Charlot, 2010, vol. 262 of IAU Symposium, pp. 362-363.

13. H. Ritter, and U. Kolb, A\&A 404, 301-303 (2003), arXiv: astro-ph/ 0301444. 